\title{
Dyspnoea modifies the recognition of fearful expressions by healthy humans
}

\begin{abstract}
To the Editor:
Brainstem neural oscillators normally generate resting breathing. Yet cortical premotor and motor respiratory-related networks can operate voluntary breathing and compensate for defective automatic respiratory drive $[1,2]$. These networks are engaged when the respiratory system load-capacity balance is compromised, typically during experimental inspiratory loading [3] that is associated with dyspnoea of the "excessive inspiratory effort" type. An emerging body of evidence suggests that respiratory-related cortical activation (and by extension the corresponding dyspnoea) can have a cognitive cost [4-6], thought to derive from competition for resources (dual-tasking) or attentional distraction. Of note, dyspnoea can also occur in response to carbon dioxide $\left(\mathrm{CO}_{2}\right)$ stimulation; in this instance, the dyspnoea is not associated with motor respiratory-related cortical activation [3] but with intense activation of limbic regions of the brain, including the insular cortex [7]. This provokes air hunger and strong emotional reactions that could interfere with the capacity to process concurrent emotions. Here we tested the hypothesis that inspiratory loading and hypercapnic stimulation affect executive and emotional processing tasks and do so in different ways.
\end{abstract}

After ethical committee approval (CPP Île-de-France 6, France), we studied 12 healthy volunteers (eight men, mean age 22.5) who gave written consent. The protocol is described in figure 1 . The subjects participated in two separate sessions in randomised order. Each session started and ended with a baseline condition. Session 1 comprised two conditions: in the dyspnoea "load" condition, subjects had to wear a nose clip and breathe through a mouthpiece against a spring-loaded valve (inspiratory threshold load, PowerBreathe ${ }^{\infty}$, Southam, UK) adjusted to obtain a rating of about $50 \%$ on a visual analogue scale anchored by "no breathing discomfort" and "intolerable breathing discomfort"; in the "motor" condition, the subjects breathed freely but had to press a pedal with their left foot in a steady rhythm. Session 2 comprised a " $\mathrm{CO}_{2}$ condition" in which dyspnoea was induced by increasing the inspiratory fraction of $\mathrm{CO}_{2}$ on demand to obtain the same ratings as during the "load" condition. In each condition, the subjects were asked to perform an executive task, i.e. an adapted version of the classical colour-word interference task (Stroop's test), and an emotion-processing task, i.e. a fear-recognition task (always in this order). During the Stroop's test, the colour words "green" and "red" were presented congruently, i.e. "green" typed in green and "red" typed in red, or incongruently, i.e. "green" typed in red and vice versa. The subjects had to judge the colour in which the word was printed as fast as possible. During the fear-recognition task, 10 versions of 18 faces (nine male, nine female; Karolinska Directed Emotional Faces [8]) ranging from "neutral" to "intensely fearful" were presented to the subjects, who were required to decide as fast as possible whether there was an expression of fear. The stimuli were presented using E-Prime ${ }^{\varpi}$ (Psychology Software Tools, Sharpsburg, PA, USA). Statistical analyses were performed with MATLAB ${ }^{\oplus}$ (Statistics and Machine Learning Toolbox; significance threshold 0.05). For Stroop data, accuracy was analysed using logistic mixed-effect regression and reaction times were analysed using linear mixed-effect regression, with two fixed categorical factors (congruence and experimental condition) and their interaction, one fixed continuous factor (time, encoded as the number of trials already performed, to account for putative learning and fatigue effects) and one random factor (intercept by subject). After encoding emotional judgement as a binary variable ( 0 for "neutral" faces, 1 for "emotional" faces), fear-recognition data were analysed using a logistic mixed-effect regression with one categorical factor (experimental condition), two

@ERSpublications

Do respiratory diseases alter emotional interactions? Becoming dyspnoeic distorts our identification of others' fear http://ow.ly/JkxF30hJGFH

Cite this article as: Vinckier F, Morélot-Panzini C, Similowski T. Dyspnoea modifies the recognition of fearful expressions by healthy humans. Eur Respir J 2018; 51: 1702253 [https://doi.org/10.1183/ 13993003.02253-2017]. 


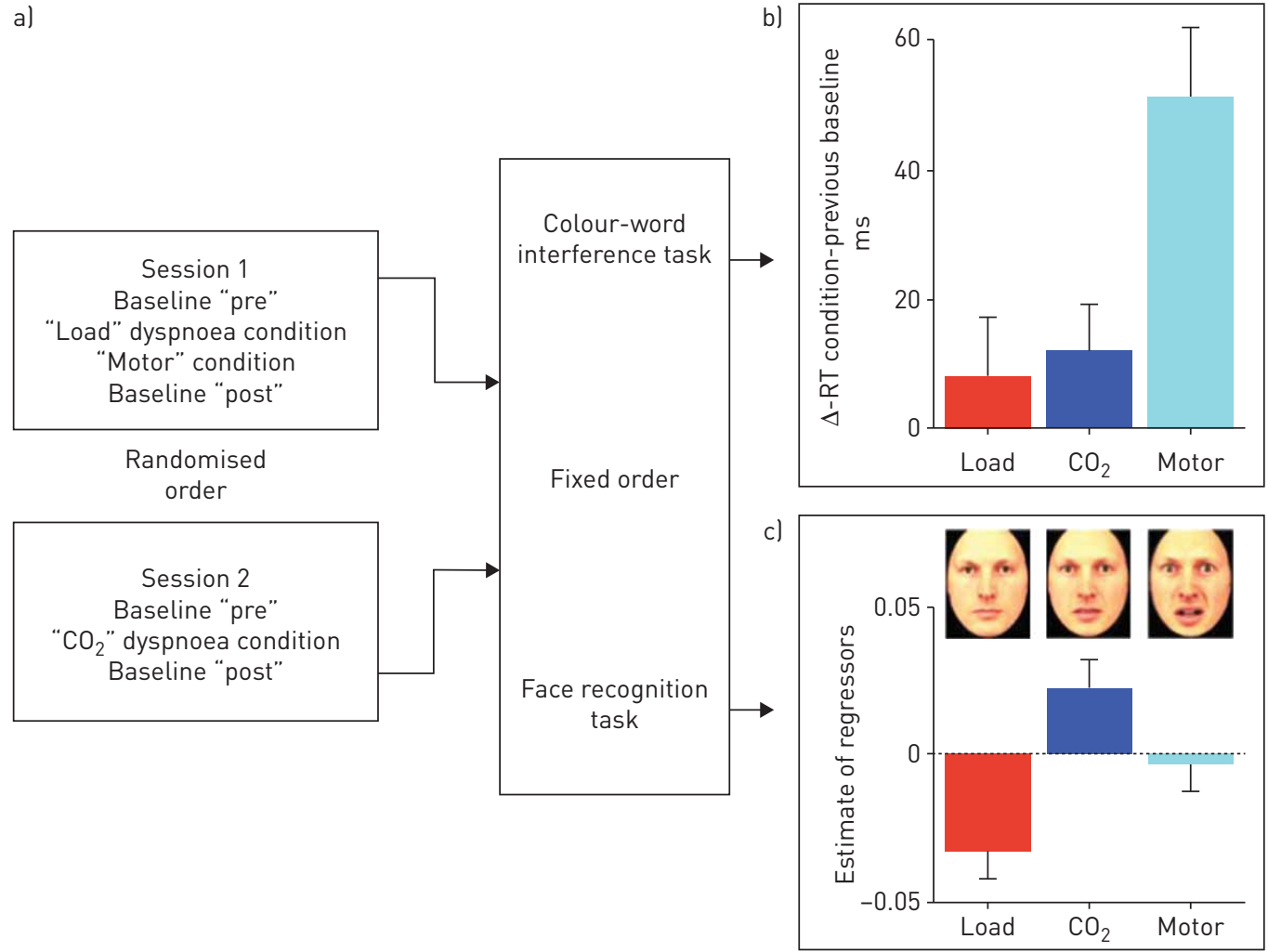

FIGURE 1 a) Schematic representation of the experimental protocol ("load": inspiratory threshold loading to induce dyspnoea; "motor": motor dual task imposed on the subjects during the behavioural tasks; " $\mathrm{CO}_{2}$ ": carbon dioxide stimulation to induce dyspnoeal. For the colour-word interference task, each trial started with a 250 ms fixation cross, which was replaced by a colour word leither "vert" or "rouge", respectively green and red in French), presented centrally in Courier New 20-point font. After 200 ms, the word was replaced by a fixation cross for $1100 \mathrm{~ms}$. In the congruent condition, the word was printed in the congruent colour le.g. "green" printed in green). In the incongruent condition, the colour word was printed in the incongruent colour (e.g. "green" printed in red). Subjects were instructed to maintain their gaze on the fixation cross and were asked to judge the colour in which the word was printed as quickly as possible. The answer was recorded during the 1120 ms but the subject was asked to answer faster if he took more than $800 \mathrm{~ms}$ to answer. Subjects had no other feedback on their performance. Each block included 80 congruent trials and 80 incongruent trials (40 "red" and 40 "green" in each condition) in a randomised order. During the fear-recognition task, each trial started with a 250 ms fixation cross, which was replaced by a face, centred on fixation. After $200 \mathrm{~ms}$, the word was replaced by a fixation cross for $1100 \mathrm{~ms}$. Subjects were instructed to maintain their gaze on the fixation cross and were required to decide whether or not there was an expression of fear on these faces. The answer was recorded during the $1300 \mathrm{~ms}$ but the subject was asked to answer faster if he took more than 1100 ms to answer. Subjects had no other feedback on their performance. In each block, each face was presented once. b) Effects of the respiratory and motor conditions on the difference in reaction times as recorded at the end of the baseline condition and during the respiratory and motor conditions (congruent and incongruent stimulation aggregated); there was no significant difference between "load" and " $\mathrm{CO}_{2}$ ", but the differences between "load" and "motor" and between " $\mathrm{CO}_{2}$ " and "motor" were both significant ( $p=0.001$ and $p=0.0001$, respectively). c) Examples of faces shown to the subjects during the fear-recognition task and results expressed in terms of the estimates of the regressors (mixed-effect logistical regression). The negative value during "load" indicates that the subjects recognised fewer faces as fearful, the opposite being true for the positive values during " $\mathrm{CO}_{2}$ ". There was no difference between the "motor" condition and baseline. RT: reaction time.

continuous factors (intensity of fear valence and time) and two random factors (intercept by subject and face identity).

Regarding the Stroop task, overall accuracy was very high (about 98\%) and slightly decreased with time $(\mathrm{t}=-3.3, \mathrm{p}<0.001)$, with no other significant effect. Reaction times were higher during incongruent presentation $(\mathrm{t}=3.7, \mathrm{p}<0.001)$ and decreased with time $(\mathrm{t}=-9.6, \mathrm{p}<0.001)$, compatible with a speedaccuracy trade-off. Reaction times were higher than at baseline in all conditions (all $\mathrm{p}<0.001$ ), without any interaction between congruence and session. Increases in reaction times (between the baseline "pre" condition and the experimental condition itself) were significantly more marked during the "motor" condition $(+51 \mathrm{~ms})$ than during the "load" (+8 ms) or " $\mathrm{CO}_{2}$ " (+12 ms) conditions, without a significant difference between the "load" and " $\mathrm{CO}_{2}$ " conditions (figure 1). 
Regarding fear recognition, there was a strong valence effect (fearful faces more often categorised as fearful, $\mathrm{t}=72.1, \mathrm{p}<0.001$ ), validating the scale construction. There was also a positive effect of time (the more the subjects saw faces, the more they judged them fearful; $\mathrm{t}=4.3, \mathrm{p}<0.001)$. Critically, faces were less often categorised as fearful during the "load" condition $(\mathrm{t}=-3.7, \mathrm{p}<0.001)$ and more often categorised as fearful during the " $\mathrm{CO}_{2}$ " condition ( $\left.\mathrm{t}=2.5, \mathrm{p}=0.01\right)$. No effect was noted during the "motor" condition (figure 1).

In this exploratory study, the colour-word interference test was not perturbed by dyspnoea whereas it was markedly impaired by the motor task. The " $\mathrm{CO}_{2}$ " dyspnoea condition was not expected to activate the premotor and motor respiratory-related cortical network and therefore not expected to induce a dual-tasking effect; this suggests that dyspnoea did not have a strong-enough attentional effect to disrupt the behavioural task. In the case of the "load" condition, the lack of interference with Stroop's test contrasts with the previously observed deterioration of a motor executive test in similar conditions [5]. This could stem from a lack of activation of the respiratory-related network (or from insufficient activation to produce a dual-tasking effect), or from a rapid cortical automatisation of the respiratory task [9]. Of note, hyperventilation does not deteriorate Stroop performances [10] even though it activates cortical respiratory networks [1]. Strikingly, in our study both types of dyspnogenic stimulation had an impact on fear recognition, which was blunted by inspiratory loading and sharpened by $\mathrm{CO}_{2}$ stimulation (figure 1). Although many layers of evidence connect fear and dyspnoea [11-13], the fact that dyspnoea can modify the capacity of individuals to recognise fear in others has never been described before. Our observations are coherent with the modulation of the neural processing of emotional pictures by perceived and anticipated respiratory threats that has been described by means of event-related potentials [14]. We acknowledge that our data are descriptive and preliminary: they need corroboration, mechanistic studies, and translation from experimental to clinical dyspnoea. Yet they could form the basis of a novel vision of the impact of chronic respiratory diseases on the life of concerned patients. It is important to emphasise that the production and recognition of facial expressions are key factors of emotional processing, itself a major determinant of human behaviour and way of life. Depression, common in patients with chronic respiratory diseases, produces negative biases in emotional processing that can be documented using fear-recognition tasks similar to the one we used [15] and that are reversed by antidepressant drugs before any self-report of mood improvement [15]. Emotional processing also has a fundamental societal function. Its deterioration is a determinant of social isolation (also common in patients with chronic respiratory diseases) or social deviance and its improvement can correct these phenomena [16]. We submit that our results have the potential to open novel research avenues: questions like "do patients with chronic respiratory diseases have trouble identifying the emotions of others?", "do certain types of dyspnoea constitute particular risk factors for depression through their impact on emotional processing" and "can therapeutic interventions correct emotional processing?" will be fascinating to investigate clinically but also regarding the anthropology of chronic respiratory diseases.

Fabien Vinckier ${ }^{1,2}$, Capucine Morélot-Panzini ${ }^{3,4}$ and Thomas Similowski ${ }^{3,4}$

${ }^{1}$ Département de psychiatrie, Service Hospitalo-Universitaire, Centre Hospitalier Sainte-Anne, Paris, France. ${ }^{2}$ Université Paris Descartes, Sorbonne Paris Cité, Laboratoire de Physiopathologie des Maladies Psychiatriques, Centre de Psychiatrie et Neurosciences, UMR S894, Paris, France. ${ }^{3}$ Sorbonne Université, INSERM, UMRS1158 Neurophysiologie respiratoire expérimentale et clinique, Paris, France. ${ }^{4} \mathrm{AP}-\mathrm{HP}$, Groupe Hospitalier Pitié-Salpêtrière Charles Foix, Service de Pneumologie et Réanimation Médicale (Département 'R3S'), Paris, France.

Correspondence: Thomas Similowski, Hopitaux Universitaires Pitie Salpetriere-Charles Foix, Service de Pneumologie, 47-83 Boulevard de l'Hôpital, Paris 75013, France. E-mail: thomas.similowski@psl.aphp.fr

Received: Oct 312017 | Accepted after revision: Dec 192017

Conflict of interest: C. Morélot-Panzini has received personal fees from AstraZeneca, Chiesi, Home Perf, Novartis, Philips and ADEP assistance, outside the submitted work. T. Similowski has received personal fees from AstraZeneca, Boerhinger Ingelheim France, GSK, Lungpacer Inc., TEVA, Chiesi, Pierre Fabre and Invacare, and personal fees and non-financial support from Novartis, outside the submitted work; in addition, he has a patent for a "brain-ventilator interface to improve the detection of dyspnoea" licensed to Air Liquide Medical Systems and MyBrainTechnology.

\section{References}

1 Dubois M, Chenivesse C, Raux M, et al. Neurophysiological evidence for a cortical contribution to the wakefulness-related drive to breathe explaining hypocapnia-resistant ventilation in humans. J Neurosci 2016; 36: 10673-10682.

2 Tremoureux L, Raux M, Hudson AL, et al. Does the supplementary motor area keep patients with Ondine's curse syndrome breathing while awake? PLoS One 2014; 9: e84534.

3 Raux M, Straus C, Redolfi S, et al. Electroencephalographic evidence for pre-motor cortex activation during inspiratory loading in humans. J Physiol 2007; 578 (Pt 2): 569-578.

4 Allard E, Canzoneri E, Adler D, et al. Interferences between breathing, experimental dyspnoea and bodily self-consciousness. Sci Rep 2017; 7: 9990. 
5 Nierat MC, Demiri S, Dupuis-Lozeron E, et al. When breathing interferes with cognition: experimental inspiratory loading alters timed up-and-go test in normal humans. PLoS One 2016; 11: e0151625.

6 Sharman M, Gallea C, Lehongre K, et al. The cerebral cost of breathing: an FMRI case-study in congenital central hypoventilation syndrome. PLoS One 2014; 9: e107850.

7 Banzett RB, Mulnier HE, Murphy K, et al. Breathlessness in humans activates insular cortex. Neuroreport 2000; 11: $2117-2120$.

8 Lundqvist D, Flykt A, Öhman A. The Karolinska Directed Emotional Faces - KDEF. CD ROM from Department of Clinical Neuroscience, Psychology section, Karolinska Institutet, ISBN 91-630-7164-9. 1998.

9 Raux M, Tyvaert L, Ferreira M, et al. Functional magnetic resonance imaging suggests automatization of the cortical response to inspiratory threshold loading in humans. Respir Physiol Neurobiol 2013; 189: 571-580.

10 Van Diest I, Stegen K, Van de Woestijne KP, et al. Hyperventilation and attention: effects of hypocapnia on performance in a stroop task. Biol Psychol 2000; 53: 233-252.

11 Feinstein JS, Buzza C, Hurlemann R, et al. Fear and panic in humans with bilateral amygdala damage. Nat Neurosci 2013; 16: 270-272.

12 Pappens M, Van den Bergh O, De Peuter S, et al. Defense reactions to interoceptive threats: a comparison between loaded breathing and aversive picture viewing. Biol Psychol 2010; 84: 98-103.

13 Ziemann AE, Allen JE, Dahdaleh NS, et al. The amygdala is a chemosensor that detects carbon dioxide and acidosis to elicit fear behavior. Cell 2009; 139: 1012-1021.

14 Juravle G, Reicherts $P$, Riechmann-Weinstein $M$, et al. Neural responses to affective pictures while anticipating and perceiving respiratory threat. Psychophysiology 2017; 54: 182-192.

15 Pringle A, Harmer CJ. The effects of drugs on human models of emotional processing: an account of antidepressant drug treatment. Dialogues Clin Neurosci 2015; 17: 477-487.

16 Hubble K, Bowen KL, Moore SC, et al. Improving negative emotion recognition in young offenders reduces subsequent crime. PLoS One 2015; 10: e0132035. 